\title{
ROTDR signal enhancement via deep convolutional denoising autoencoders trained with domain randomization
}

I. Laarossi, A. Pardo Franco, O. M. Conde, M. A. Quintela, José Miguel López-Higuera

I. Laarossi, A. Pardo Franco, O. M. Conde, M. A. Quintela, José Miguel López-Higuera, "ROTDR signal enhancement via deep convolutional denoising autoencoders trained with domain randomization," Proc. SPIE 11199, Seventh European Workshop on Optical Fibre Sensors, 111993N (14 October 2019); doi: 10.1117/12.2540012

Event: Seventh European Workshop on Optical Fibre Sensors, 2019, Limassol, Cyprus 


\title{
ROTDR signal enhancement via deep convolutional denoising autoencoders trained with domain randomization
}

\author{
I. Laarossi*a , A. Pardo ${ }^{\mathrm{a}, \mathrm{c}}$, O. M. Conde ${ }^{\mathrm{a}, \mathrm{b}, \mathrm{c}}$, M.A. Quintela ${ }^{\mathrm{a}, \mathrm{b}, \mathrm{c}}$ and J. M. López-Higuera ${ }^{\mathrm{a}, \mathrm{b}, \mathrm{c}}$ \\ ${ }^{a}$ Photonics Engineering Group, University of Cantabria, 39005 Santander, Spain; ${ }^{\mathrm{b}}$ CIBER-BBN, \\ Instituto de Salud Carlos III, 28029 Madrid, Spain; ' Instituto de Investigación Sanitaria Valdecilla \\ (IDIVAL), 39011 Santander, Spain
}

\begin{abstract}
In this work, a deep convolutional adaptive filter is proposed to enhance the performance of a Raman based distributed temperature sensor system by the application of domain randomization methods for its training. The improvement of the signal-to-noise ratio in the Raman backscattered signals in the training process and translation to a real scenario is demonstrated. The ability of the proposed technique to reduce signal noise effectively is proved independently of the sensor configuration and without degradation of temperature accuracy or spatial resolution of these systems. Moreover, using single trace to noise reduction in the ROTDR signals accelerates the system response avoiding the employment of many averages in a unique measurement.
\end{abstract}

Keywords: Spontaneous Raman Scattering, fiber optic sensors, Raman Distributed Temperature sensors, Optical-timedomain-reflectometry, gold-coated fibers, domain randomization, neural networks, adaptive filter.

\section{INTRODUCTION}

In recent years, Spontaneous Raman based optical fiber distributed temperature sensors (DTS) have been an intense area of research to provide solutions to real and unresolved problems in numerous industrial applications and have found a practical and economic applicability in many fields and industrial scenarios: oil and gas sector, thermal control of power lines, pipeline leakage detection, etc. [1]. The known advantages offered by these sensors [2] are increasingly driving the use of this technology against traditional discrete temperature sensors, providing the ability to sense tens of kilometers with a high spatial and temperature resolutions and exhibiting immunity to electromagnetic fields in harsh environments [3].

The most common Raman DTS systems employ the optical time domain reflectometry (OTDR) technique to measure the distributed temperature profile along the fiber, exploiting the high sensitivity to temperature of the spontaneous AntiStokes Raman component. For this propose, short pulses are coupled into the fiber (their duration determines the spatial resolution of the measurements), and as the probe light propagates along it, the two components of the Raman scattering (Stokes and Anti-Stokes) are generated. A small portion of both components, containing information about losses and temperature along the entire fiber, is guided back to the launching end and detected by photodiodes (usually avalanche photodiodes, APDs). Knowing the time elapsed between the pulse launched and the arrival time of the returning photons, it is possible to calculate the position $\mathrm{z}$ in the fiber where the scattering has occurred.

Usually the ratio of the Anti-Stokes to Stokes intensities is used to estimate the fiber temperature, avoiding possible errors resulting from, for example, variations of fiber losses, such as losses produced by the splices. The major drawback of the use of this method is the low powers of the Raman backscattered detected signals (up to $70 \mathrm{~dB}$ below the power of the injected light [4]). This fact forces the use of high pulse powers (without reaching the Raman stimulated scattering threshold power) and many averages to obtain an acceptable value of one of the most critical parameters for determining the performance of Raman DTS systems: the signal-to-noise ratio (SNR). This high number of averages for each measurement leads to a slow response in these systems.

*Ismail Laarossi; 1aarossii@unican.es

Seventh European Workshop on Optical Fibre Sensors, edited by Kyriacos Kalli, Gilberto Brambilla, Sinead O'Keeffe Proc. of SPIE Vol. 11199, 111993N · C 2019 SPIE · CCC code: 0277-786X/19/\$21 · doi: 10.1117/12.2540012 
To overcome this problem, recent works present other alternatives, such as, for example, the use of deep learning systems, and more precisely, auto-encoders to enhance the ROTDR signals [5]. This method increases the SNR of the ROTDR signals by $10-15 \mathrm{~dB}$, as long as multiple traces are captured under only white Gaussian noise (AWGN, Additive White Gaussian Noise) and the configuration of the channel does not change. However, in cases where real-time measurements with high spatial resolution are needed or when the channel configuration can be change throughout the sensor life, this technique may not be suitable. Other methods, such as, for example, ARMA filters (AutoRegressive-Moving-Average) or wavelets, improve the SNR of the backscattered signals using unidimensional data (1D), but affecting the spatial resolution of the ROTDR signals [6,7].

In this article, we present a new technique for enhance the ROTRD signals, using an adaptive deep filter, of a completely convolutional type. This method analyzes a single trace at a time (increasing the system response) and tries to improve the SNR of the backscattered signals without distorting the traces detected in the Raman based distributed temperature sensors.

\section{MATERIALS AND METHODS}

\subsection{Experimental Setup}

In order to train the model presented in this work, a variety of simulated environments is generated using the theoretical equations that model the Stokes and Anti-Stokes backscattered signals in the optical fibers [8]:

$$
\begin{aligned}
& P_{A S}(z, T)=\frac{C_{A S}}{e^{\frac{h \Delta v}{k T(z)}}-1} e^{-\int_{0}^{z}\left(\alpha_{A S}(\epsilon)+\alpha_{p}(\epsilon)\right) d \epsilon} \\
& P_{S}(z, T)=\frac{C_{S}}{1-e^{-\frac{h \Delta v}{k T(z)}}} e^{-\int_{0}^{z}\left(\alpha_{S}(\epsilon)+\alpha_{p}(\epsilon)\right) d \epsilon}
\end{aligned}
$$

where, $z$ is the distance along the fiber, $T(z)$ is the absolute temperature and $\alpha_{A S}, \alpha_{S}$ and $\alpha_{p}$ are the attenuations at AntiSotkes, Stokes and probe light wavelengths, respectively. $C_{A S}$ and $C_{S}$ are dimensionless constants are dimensionless constants depending on parameters such us the power of the input pump signal, the response of the photodetectors, etc. Finally, $k$ is the Boltzmann's constant, $h$ the Planck's constant and $\Delta v$ the separation between Raman anti-Stokes/Stokes and probe light frequencies.

In other hand, to demonstrate the capability of the model used to enhance the ROTDR signals in a real scenario, the experimental set-up shown in Figure 1 has been developed. The anti-Stokes and Stokes signal traces have been obtained through a Raman Distributed Temperature Sensor (RDTS) unit with a spatial resolution of $0.5 \mathrm{~m}$ (corresponding to a pulses width $5 \mathrm{~ns}$ duration). The source of the RDTS unit is provided by a laser operating at $1064 \mathrm{~nm}$ with an average output power of $17 \mathrm{~mW}$.

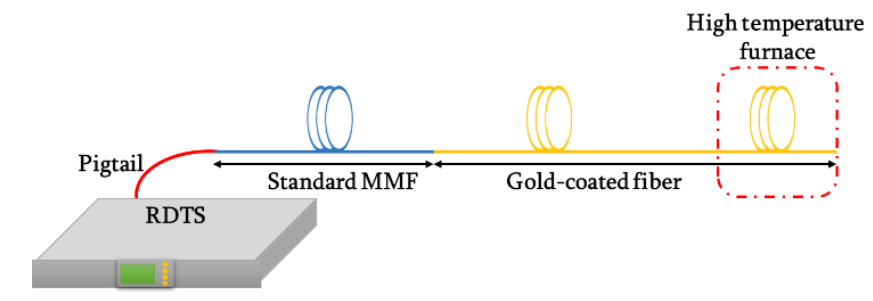

Figure 1: Schematic diagram of the RTDS system used in the experimental tests.

The fiber under test (FUT) used in set-up consists in $100 \mathrm{~m}$ of a standard graded-index multimode fiber (MMF-50/125 $\mu \mathrm{m}$ ) and $150 \mathrm{~m}$ of a pure silica core gold-coated fiber. The last $20 \mathrm{~m}$ of the gold-coated fiber was inserted into a furnace chamber and the temperature was modified between room temperature $\left(20{ }^{\circ} \mathrm{C}\right.$ approximately $)$ and $450{ }^{\circ} \mathrm{C}$ with steps of $25{ }^{\circ} \mathrm{C}$.

\subsection{Domain randomization}

To obtain an adaptive filter that can operate for every possible scenario and setup, it is fundamental to present to the model a vast amount $\left(\sim 10^{6}\right)$ of possible setup configurations. Since this is not possible in practice, synthetic signals must be 
generated. This learning procedure is called domain randomization and is especially important in cases where robustness and immediacy are required [9]. In the case of ROTDR signals, the following setup characteristics are randomized: (a) fiber splices and their positions in space, as well as their insertion losses (from 0.1 to $3 \mathrm{~dB}$ ); (b) hotspot zones throughout the sensor, their length and their positions in space; (c) the temperature of said zones within a reasonable range (from 0 to $800^{\circ} \mathrm{C}$ ); and (d) noise variance, in a range similar to those shown by the real ROTDR traces. These parameters are randomized for every synthesized trace. Each training vector consists, then, of an artificial ROTDR trace without noise $\left(y_{i}\right)$, and the same trace with added noise $\left(x_{i}\right)$. For each iteration during learning, 100 random traces are generated, and used to train the model via mini-batch training descent.

\subsection{Architecture and training}

The model used is a fully convolutional denoising autoencoder (DAE), with 24 ELU layers (12 for coding and 12 for decoding). The bottleneck is 15 neurons, through which only relevant information must pass. Although domain randomization is used, as a precaution all convolutional layers except the output layer also implement dropout with probability $p=0.1$. No max pooling layers are used. The output layer of the filter is a linear layer of the same dimensions as the input data. To train the model, 100-trace mini-batches and a total of $200 \cdot 10^{3}$ iterations are used, using ADAM as the core optimization algorithm (learning hyperparameter is set to $1 \cdot 10^{-5}, L=\frac{1}{2} \sum_{n=1}^{N}\left\|\widehat{y}_{i}\left(x_{i}\right)-y_{i}\right\|^{2}$ ). The selected cost function was the Mean Square Error (MSE).

\subsection{Training results}

After training, the system is able to reduce the mean square error of the complete trace to $10^{-1}(<0.1 \%)$, validation errors are maintained below training errors, showcasing resilience against overfitting. Figure 2 includes three examples of ROTDR traces of the Stokes component of the simulated sensor: the noisy semi-transparent curve is the input data, the color curve is the desired signal, and the dotted black curve is the output filter response.

The behavior of the system tends to prefer to adapt to fiber transitions and to hot-spot sections of greater length, since the function to be optimized (Equation 3) forces these sections to predominate point sensors. It must be noted here that the system is correcting SNR with a single trace, and that it is filtering adaptively in space, preserving the rectangular structure of the sensors and without significantly damaging the temperature values, for any possible configuration. If it is the same sensor, the system would have much easier to adapt to that environment, where splices, hot-spot sections and losses are always under the same conditions and the only independent variable is the temperature variation.
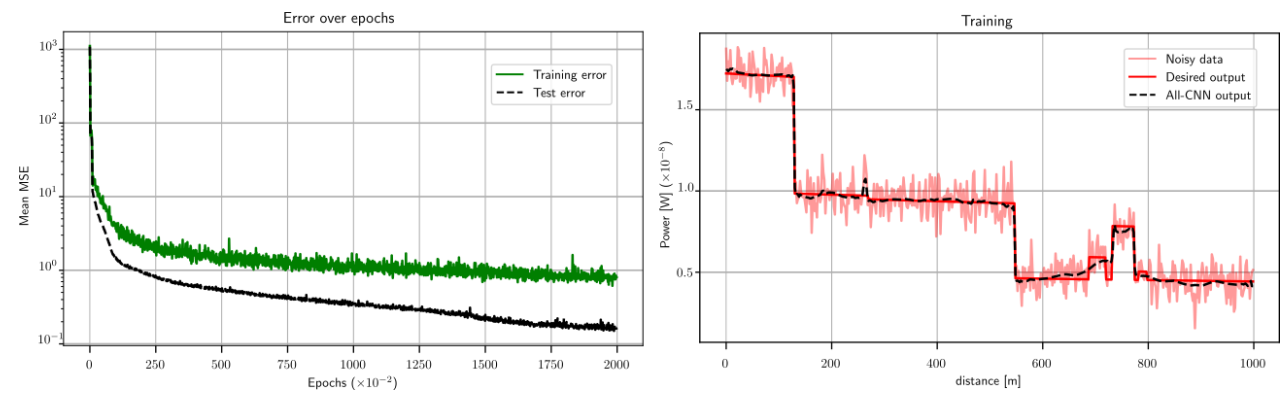

Fig. 2: Adaptive filter behavior: (left) validation/test errors (in black) remain below training losses (in green) during the complete training process, thus avoiding overfitting; (right) an example of a randomized OTDR scenario during training.

\subsection{Practical results}

An example of practical translation of the adaptive filter into a real-world scenario is shown in Figure 3. The scenario presented in Section 2.1 has a hot-spot section at the final termination of the fiber, under a considerable amount of noise, along $250 \mathrm{~m}$ of fiber. The proposed filter adapts well to system temperature variations, which have never been presented to the system until now.

It is important to highlight that this adaptive filter, with the exception of hot zones of reduced dimensions (in the order of the size of convolutional filters), is successful in attempting to minimize spatial distortion, despite being based on the convolution operator. Therefore, it can be very useful if real-time temperature estimation is required ( 30 estimates / s) with acceptable spatial resolution. 

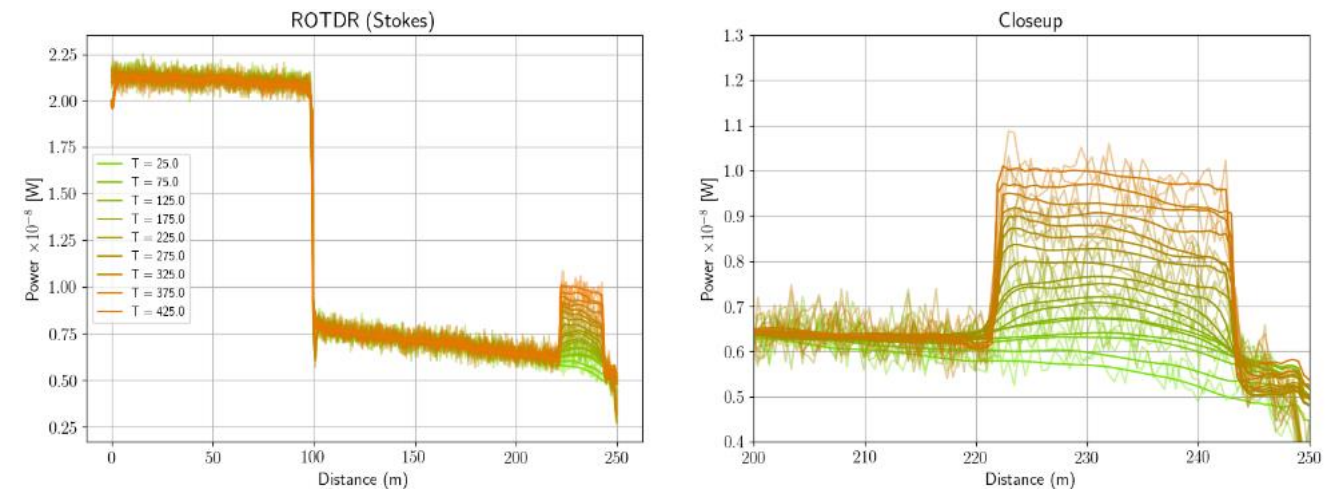

Fig. 3: Behavior of the adaptive filter after applying it over a real signal with temperature variations. The values along the sensor undergo a filtering that is free of damage in the spatial domain as shown on the right subplot showing a closeup of the measurement. Input noisy measurements (semitransparent lines) are compared with filtered measurements (solid lines).

\section{CONCLUSION}

The present contribution demonstrates the single-shot behavior of a fully convolutional autoencoder to filter ROTDR signals independently of system configuration. The adaptative filter is trained via domain randomization, using synthetic ROTDR signals, to remove signal noise effectively and without affecting the system parameters. The translational potentials of the method were empirically verified, enhancing real ROTDR measurement with rapid temporal response while still maximizing spatial resolution.

\section{ACKNOWLEDGEMENTS}

This work has been supported by Spanish CICYT (TEC2016-76021-C2-2-R), by ISCIII (DTS17-00055, INTRACARDIO) co-funded by EU-FEDER FUNDS and by the Spanish Ministry of Education, Culture and Sports through FPU16/05705.

\section{REFERENCES}

[1] Adachi, S., "Distributed optical fiber sensors and their applications," SICE Annual Conference. IEEE. p. 329 333, 2008.

[2] López-Higuera, J. M., (ed.). "Handbook of optical fibre sensing technology,” Wiley, 2002.

[3] Williams, G. R., Brown, G., Hawthorne, W., Hartog, A. H., \& Waite, P. C., "Distributed temperature sensing (DTS) to characterize the performance of producing oil wells," Industrial Sensing Systems. International Society for Optics and Photonics, 4202, pp. 39-55, 2000.

[4] Soto, M. A, Signorini, T., Lazzeri, A., Baronti, A., Roncella, F., Di Pasquale, R., "Raman-based distributed temperature sensor with $1 \mathrm{~m}$ spatial resolution over $26 \mathrm{~km}$ SMF using low-repetition-rate cyclic pulse coding," Optics letters, 36, no 13, p. 2557-2559, 2011.

[5] Wu, H., Zhao, C., Liao, R., Chang, Y., Tang, M., "Performance enhancement of ROTDR using deep convolutional neural networks. Optical Fiber Sensors. Optical Society of America," p. TuE16, 2018.

[6] Wang, Z., C4, J., Zhang, S., Luo, S., Jia, C., Sun, Jiang, B., S., Liu, Y., Liu, X., LV, G., and Liu, Z., “Application of Wavelet Transform Modulus Maxima in Raman Distributed Temperature Sensors," Photonic Sensors, 4, No. 2, pp. 142-146, 2014.

[7] Saxena, M. K., Raju, S. D. V. S. J., Arya, R., Pachori, R. B., Ravindranath, S. V. H., Kher, S., and Oak, S. M., "Raman optical fiber distributed temperature sensor using wavelet transform based simplified signal processing of Raman backscattered signals," Optics \& Laser Technology, 65, pp. 14-24, 2015.

[8] Hausner, M. B., Suárez F., Glander, K. E., Giesen, N. V. D., Selker, J.S. and Tyler, S.W., "Calibrating singleended fiber-optic Raman spectra distributed temperature sensing data," Sensors. Vol 11, pp. 10859-10879, 2011.

[9] Tobin, J., Fong, R., Ray, A., Schneider, J, Zaremba, W. and Abbeel, P., "Domain Randomization for Transferring Deep Neural Networks from Simulation to the Real World," IEEE/RSJ International Conference on Intelligent Robots and Systems (IROS 2017), Subjects: Robotics (cs.RO); Machine Learning (cs.LG), 2017. 